\title{
General Fifth M-Zagreb Polynomials of Benzene Ring Implanted in the P-Type-Surface in 2D Network
}

\author{
Prosanta Sarkar $^{1, *}$ (D), Anita Pal ${ }^{1}$ \\ 1 National Institute of Technology Durgapur, India \\ * Correspondence: prosantasarkar87@gmail.com;
}

Scopus Author ID 57203859853

Received: 1.05.2020; Revised: 26.05.2020; Accepted: 27.05.2020; Published: 1.06.2020

\begin{abstract}
Constitutional formulae of molecules are molecular graphs consisting of atoms as vertices and bonds between them represented as edges. The various physical, chemical, and biological properties of molecules are dependent on their molecular structures. The molecular structure is most important, not only to chemists but also to all scientists. The molecular structure descriptors or topological indices of molecules are a mathematical number or a set of selected invariants of matrices that are used to Quantitative Structure-Activity (-Property) Relationships (QSAR/QSPR) studies. In this paper, we computed some new degree-based topological indices of benzene ring implanted in the P-type-surface in the 2D network and its line subdivision of graph.
\end{abstract}

Keywords: General fifth M-Zagreb polynomials; Third Zagreb polynomial; Various degree-based topological indices; Benzene ring implanted in the P-type-surface network.

(C) 2020 by the authors. This article is an open-access article distributed under the terms and conditions of the Creative Commons Attribution (CC BY) license (https://creativecommons.org/licenses/by/4.0/).

\section{Introduction}

In chemical graph theory, a molecular graph is a representation of the structural formula of a molecule in the form of graph structure, where atoms are represented as vertices and bonds of atoms are considered as edges. A molecular graph is a finite, simple, and connected graph. Basically, a graph is denoted by $G \equiv(V(G), E(G))$, where $V(G)$ denote the vertex set and $E(G)$ denote the edge set of $G$, respectively. The number of vertices in $G$ is called the order of $\mathrm{V}(\mathrm{G})$ and is denoted by $|\mathrm{V}(\mathrm{G})|$. The number of edges in $\mathrm{G}$ is called the order of $E(\mathrm{G})$ and is denoted by $|E(G)|$. A subdivision graph $S(G)$ is derived from $\mathrm{G}$ by inserting a new vertex into each edge of $\mathrm{G}$. The line graph $L(G)$, is the graph whose vertices are the edges of $\mathrm{G}$ and two vertices e, $f \in L(G)$ are connected if and only if they share a common vertex in $G$. The degree of a vertex $a \in G$, is the number of neighbor vertices of $a$ and is denoted by $d_{G}(a)$. The sum of the degrees of neighbor vertices of any vertex $a$ in $G$ is denoted by $S_{G}(a)$. A molecular structure descriptor or a topological index is a real-valued function $f: G \rightarrow \mathbb{R}$, which maps each molecular graph to certain real numbers, and it remains invariant under graph isomorphism. In the past two decades, a large number of topological indices have been considered by some eminent researchers to utilized for relationship examination in chemistry, pharmacology, toxicology, and ecological science [1-7]. Nowadays, these indices are extensively used in building quantitative structure-property relationship (QSPR), quantitative structure-activity relationship (QSAR), and quantitative structure toxicity relationship (QSTR) [8-11]. In this work, we compute various degree-based topological indices such as fifth $M-$ Zagreb indices 
and their polynomials, fifth hyper $M$-Zagreb indices and their polynomials, general fifth $M$-Zagreb indices and their polynomials, third Zagreb index or fifth $M_{3}$-Zagreb index and it is polynomial for a benzene ring implanted in $P$-type surface structure.

\section{Materials and Methods}

2.1. Various degree-based topological indices.

In [12], Graovac et al. first introduced fifth $M$-Zagreb indices in 2011. They defined these indices as

$$
\mathrm{M}_{1} \mathrm{G}_{5}(\mathrm{G})=\sum_{a b \in E(G)}\left(S_{G}(a)+S_{G}(b)\right)
$$

and

$$
\mathrm{M}_{2} \mathrm{G}_{5}(\mathrm{G})=\sum_{a b \in E(G)}\left(S_{G}(a) S_{G}(b)\right) .
$$

In 2017, V.R. Kulli [13], generalized these indices as

and

$$
\mathrm{M}_{1}^{\alpha} \mathrm{G}_{5}(\mathrm{G})=\sum_{a b \in E(G)}\left(S_{G}(a)+S_{G}(b)\right)^{\alpha}
$$

$$
\mathrm{M}_{2}^{\alpha} \mathrm{G}_{5}(\mathrm{G})=\sum_{a b \in E(G)}\left(S_{G}(a) S_{G}(b)\right)^{\alpha} .
$$

In the same paper [13], he also introduced fifth hyper $M$-Zagreb indices as

and

$$
\mathrm{HM}_{1} \mathrm{G}_{5}(\mathrm{G})=\sum_{a b \in E(G)}\left(S_{G}(a)+S_{G}(b)\right)^{2}
$$

$$
\mathrm{HM}_{2} \mathrm{G}_{5}(\mathrm{G})=\sum_{a b \in E(G)}\left(S_{G}(a) S_{G}(b)\right)^{2} .
$$

In [13], he also defined a new version of third Zagreb index or fifth $M_{3}-$ Zagreb index as

$$
M_{3} G_{5}(G)=\sum_{a b \in E(G)}\left|S_{G}(a)-S_{G}(b)\right| .
$$

Followed by these indices in his paper [13], he defined Zagreb polynomials as follows:

The fifth $M-$ Zagreb polynomials are defined as

$$
\mathrm{M}_{1} \mathrm{G}_{5}(\mathrm{G}, \mathrm{x})=\sum_{a b \in E(G)} x^{\left(S_{G}(a)+S_{G}(b)\right)}
$$

and

$$
\mathrm{M}_{2} \mathrm{G}_{5}(\mathrm{G}, \mathrm{x})=\sum_{a b \in E(G)} x^{\left(S_{G}(a) S_{G}(b)\right)}
$$

where, $x$ is a variable. Fifth hyper $M$-Zagreb polynomials are defined as

and

$$
\mathrm{HM}_{1} \mathrm{G}_{5}(\mathrm{G}, \mathrm{x})=\sum_{a b \in E(G)} x^{\left(S_{G}(a)+S_{G}(b)\right)^{2}}
$$

$$
\mathrm{HM}_{2} \mathrm{G}_{5}(\mathrm{G}, \mathrm{x})=\sum_{a b \in E(G)} x^{\left(S_{G}(a) S_{G}(b)\right)^{2}}
$$


General fifth $M$-Zagreb polynomials are defined as

$$
\mathrm{M}_{1}^{\alpha} \mathrm{G}_{5}(\mathrm{G}, \mathrm{x})=\sum_{a b \in E(G)} x^{\left(S_{G}(a)+S_{G}(b)\right)^{\alpha}}
$$

and

$$
\mathrm{M}_{2}^{\alpha} \mathrm{G}_{5}(\mathrm{G}, \mathrm{x})=\sum_{a b \in E(G)} x^{\left(S_{G}(a) S_{G}(b)\right)^{\alpha}}
$$

where, $\alpha \in \mathbb{R}, \alpha \neq 0$ and $x$ is a variable. The new version of third Zagreb or $M_{3}-$ Zagreb polynomial is defined as

$$
\mathrm{M}_{3} \mathrm{G}_{5}(\mathrm{G}, \mathrm{x})=\sum_{a b \in E(G)} x^{\left(\left|S_{G}(a)-S_{G}(b)\right|\right)} .
$$

Some polynomials and their corresponding topological indices were studied in [14-19].

\subsection{Benzene ring implanted in P-type surface in $2 D$ network.}

The nanoscience, a period starting in 1985 , when $C_{60}$ is discovered. The branch is controlled by carbon allotropes to studying for applications in nanotechnology. Among these, nanotubes, fullerenes, graphene, diamond, and spongy nanostructures were the most studied [20-23]. In 1991, Mackay and Terrones [24], have proposed the concept of making conceivable solid carbon with three coordinated frames by tilling the infinite periodic minimal surfaces named as $P$ and $D$, which are separate space into two disjoint mazes. Later Lenosky et al. [25], has proposed conceivable three-dimensional carbon solids using $D$ tilling of 192 atoms per unit cell, a $P$ tilling of 216 atoms per unit cell. In 1992, M.O. Keeffe et al. [26], compare the energies of these proposed structures composed of six-fold, and eightfold rings happen in the proportion 2: 3, and both have cells of just 24 atoms to those of graphite and of the icosahedral fullerene $C_{60}$. They named the second structure as poly benzene is seen as more steady than $C_{60}$ energetically. Poly benzene might be depicted as a three-dimensional linkage of $C_{6}$ (benzene like) rings, thus the name poly benzene is predicted to be insulating. They manage two three dimensional frameworks of benzene, one of them is called $6.8^{2} P$ (moreover poly benzene) and has a place with space accumulate $\operatorname{Im} 3 \mathrm{~m}$, contrasting with the $P$-type surface. Generally, this structure is obtained by embeddings of the hexagon-fix in the surface of the negative ebb and flow $\mathrm{P}$ (For more about this network, we refer our reader to $[26,27]$ ). The molecular graph $G$ of benzene ring implanted in the $P$-type surface in $2 D$ network and its line subdivision graph $\mathrm{L}(\boldsymbol{S}(\mathrm{G}))$ is shown in Figure 1 and Figure 2, respectively.

\section{Results and Discussion}

In this section, we compute General fifth $M$-Zagreb polynomials and the new version of the third Zagreb polynomial of the molecular graph $G$ of a two-dimensional network of benzene ring implanted in P-type-surface and its line subdivision graph $\mathrm{L}(\boldsymbol{S}(\mathrm{G}))$. With the help of these two polynomials, we compute some other polynomials and their corresponding topological indices, which are mentioned earlier in this work. First, we compute general fifth $M$-Zagreb polynomials for $G$. The edge partition of graph $G$ based on the degree sum of neighbor vertices of end vertices of each edge, is shown in Table 1 . The total number of vertices and edges in $G$ are $24 \mathrm{hk}$ and ( $32 \mathrm{hk}-2 \mathrm{~h}-2 \mathrm{k})$, respectively. 


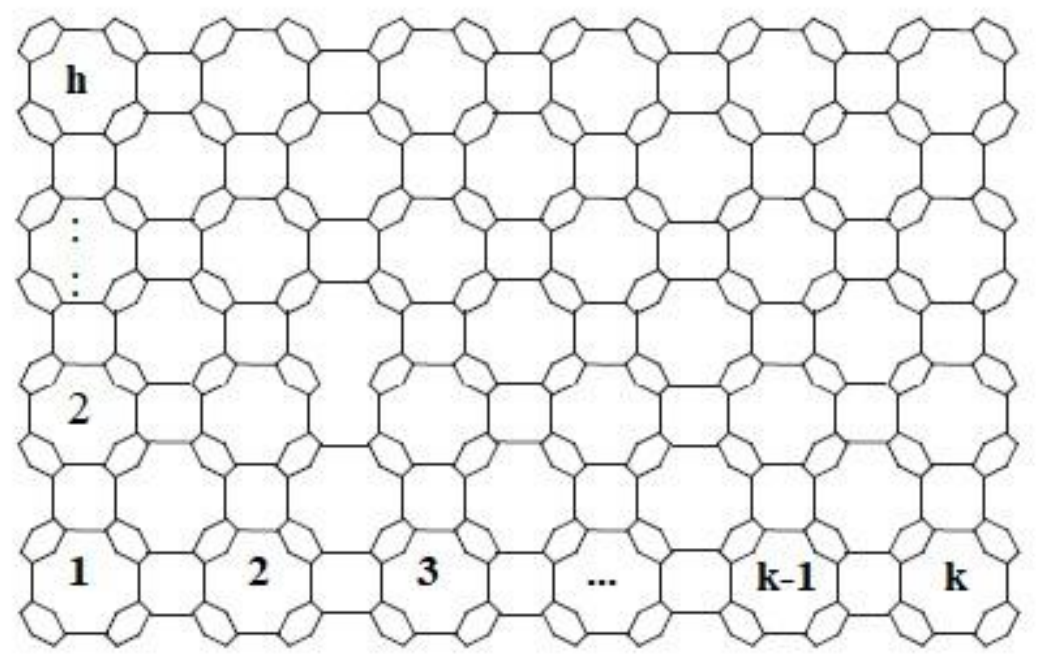

Figure 1. Two-dimensional networks of benzene ring implanted in P-type-surface

Table 1. The edge partitions with respect to the degree sum of neighbor vertices of end vertices of every edge of $G$.

\begin{tabular}{cc}
\hline$(\boldsymbol{S}(\boldsymbol{a}), \boldsymbol{S}(\boldsymbol{b})): \boldsymbol{a} \boldsymbol{b} \in \boldsymbol{E}(\boldsymbol{G})$ & Total number edges \\
\hline$(4,5)$ & 8 \\
$(5,5)$ & $(4 h+4 k-8)$ \\
$(5,7)$ & $(4 h+4 k)$ \\
$(5,8)$ & $(4 h+4 k-8)$ \\
$(6,7)$ & $(4 h+4 k)$ \\
$(6,8)$ & $(16 h k-12 h-12 k+8)$ \\
$(7,7)$ & $(2 h+2 k)$ \\
$(8,8)$ & $(16 h k-8 h-8 k)$ \\
\hline
\end{tabular}

Theorem 1. The general fifth $M_{1}-$ Zagreb polynomial of $G$ is given by $\begin{aligned} M_{1}^{\alpha} G_{5}(G, x) & =(16 h k-8 h-8 k) x^{16^{\alpha}}+(16 h k-10 h-10 k+8) x^{14^{\alpha}} \\ & +(8 h+8 k-8) x^{13^{\alpha}}+(4 h+4 k) x^{12^{\alpha}}+(4 h+4 k-8) x^{10^{\alpha}}+8 x^{9^{\alpha}}\end{aligned}$

Proof. From the definition of general fifth $M_{1}-$ Zagreb polynomial index we get,

$$
\begin{aligned}
\mathrm{M}_{1}^{\alpha} \mathrm{G}_{5}(\mathrm{G}, \mathrm{x})= & \sum_{a b \in E(G)} x^{\left(S_{G}(a)+S_{G}(b)\right)^{\alpha}} \\
= & \sum_{a b \in E_{1}(G)} x^{(4+5)^{\alpha}}+\sum_{a b \in E_{2}(G)} x^{(5+5)^{\alpha}}+\sum_{a b \in E_{3}(G)} x^{(5+7)^{\alpha}}+\sum_{a b \in E_{4}(G)} x^{(5+8)^{\alpha}} \\
& +\sum_{a b \in E_{5}(G)} x^{(6+7)^{\alpha}}+\sum_{a b \in E_{6}(G)} x^{(6+8)^{\alpha}}+\sum_{a b \in E_{7}(G)} x^{(7+7)^{\alpha}}+\sum_{a b \in E_{8}(G)} x^{(8+8)^{\alpha}} \\
= & \left|E_{1}(G)\right| x^{9^{\alpha}}+\left|E_{2}(G)\right| x^{10^{\alpha}}+\left|E_{3}(G)\right| x^{12^{\alpha}}+\left|E_{4}(G)\right| x^{13^{\alpha}} \\
& +\left|E_{5}(G)\right| x^{13^{\alpha}}+\left|E_{6}(G)\right| x^{14^{\alpha}}+\left|E_{7}(G)\right| x^{14^{\alpha}}+\left|E_{8}(G)\right| x^{16^{\alpha}} \\
= & 8 x^{9^{\alpha}}+(4 \mathrm{~h}+4 \mathrm{k}-8) x^{10^{\alpha}}+(4 \mathrm{~h}+4 \mathrm{k}) x^{12^{\alpha}}+(4 \mathrm{~h}+4 \mathrm{k}-8) x^{13^{\alpha}} \\
& +(4 \mathrm{~h}+4 \mathrm{k}) x^{13^{\alpha}}+(16 \mathrm{hk}-12 \mathrm{~h}-12 \mathrm{k}+8) x^{14^{\alpha}}+(2 \mathrm{~h}+2 \mathrm{k}) x^{14^{\alpha}} \\
& +(16 \mathrm{hk}-8 \mathrm{~h}-8 \mathrm{k}) x^{16^{\alpha}}
\end{aligned}
$$

Hence, the result follows as in equation 1. 
Corollary 1. In equation 1 , replacing $\alpha=1$ and $\alpha=2$ respectively we get the $M_{1} G_{5}(G, x)$ and $H M_{1} G_{5}(G, x)$ as follows:

i) $\quad M_{1} G_{5}(G, x)=(16 \mathrm{hk}-8 \mathrm{~h}-8 \mathrm{k}) \mathrm{x}^{16}+(16 \mathrm{hk}-10 \mathrm{~h}-10 \mathrm{k}+8) \mathrm{x}^{14}$ $+(8 \mathrm{~h}+8 \mathrm{k}-8) \mathrm{x}^{13}+(4 h+4 k) x^{12}+(4 h+4 k-8) x^{10}+8 x^{9}$,

(ii) $H M_{1} G_{5}(G, x)=(16 \mathrm{hk}-8 \mathrm{~h}-8 \mathrm{k}) \mathrm{x}^{256}+(16 \mathrm{hk}-10 \mathrm{~h}-10 \mathrm{k}+8) \mathrm{x}^{196}$

$$
+(8 \mathrm{~h}+8 \mathrm{k}-8) \mathrm{x}^{169}+(4 h+4 k) x^{144}+(4 h+4 k-8) x^{100}+8 x^{81} \text {. }
$$

Proposition 1. Differentiate the counting polynomial as shown in equation 1, with respect to $x$ at $x=1$, we get the general fifth $M_{1}$-Zagreb index as follows:

(i ) $\mathrm{M}_{1}^{\alpha} \mathrm{G}_{5}(\mathrm{G})=(16 \mathrm{hk}-8 \mathrm{~h}-8 \mathrm{k})(16)^{\alpha}+(16 \mathrm{hk}-10 \mathrm{~h}-10 \mathrm{k}+8)(14)^{\alpha}$

$$
+(8 h+8 k-8)(13)^{\alpha}+(4 h+4 k)(12)^{\alpha}+(4 h+4 k-)(10)^{\alpha}+8.9^{\alpha} .
$$

Corollary 2. In Proposition 1, replacing $\alpha=1$ and $\alpha=2$ respectively we get the $M_{1} G_{5}(G)$ and $H M_{1} G_{5}(G)$ as follows:

(i) $M_{1} G_{5}(G)=480 h k-76 h-76 k$,

(ii) $H M_{1} G_{5}(G)=7232 h k-1680 h-1680 k-584$.

Theorem 2. The general fifth $M_{2}-$ Zagreb polynomial of $G$ is given by

$$
\begin{aligned}
\mathrm{M}_{2}^{\alpha} \mathrm{G}_{5}(\mathrm{G}, \mathrm{x})= & (16 \mathrm{hk}-8 \mathrm{~h}-8 \mathrm{k}) x^{64^{\alpha}}+(2 \mathrm{~h}+2 \mathrm{k}) x^{49^{\alpha}}+(16 \mathrm{hk}-12 \mathrm{~h}-12 \mathrm{k}+8) x^{48^{\alpha}} \\
& +(4 \mathrm{~h}+4 \mathrm{k}) x^{42^{\alpha}}+(4 \mathrm{~h}+4 \mathrm{k}-8) x^{40^{\alpha}}+(4 \mathrm{~h}+4 \mathrm{k}) x^{35^{\alpha}} \\
& +(4 \mathrm{~h}+4 \mathrm{k}-8) x^{25^{\alpha}}+8 x^{20^{\alpha}}
\end{aligned}
$$

Proof. From the definition of general fifth $M_{2}$-Zagreb polynomial index we get,

$$
\begin{aligned}
\mathrm{M}_{2}^{\alpha} \mathrm{G}_{5}(\mathrm{G}, \mathrm{x})= & \sum_{a b \in E(G)} x^{\left(S_{G}(a) S_{G}(b)\right)^{\alpha}} \\
= & \sum_{a b \in E_{1}(G)} x^{(4 \times 5)^{\alpha}}+\sum_{a b \in E_{2}(G)} x^{(5 \times 5)^{\alpha}}+\sum_{a b \in E_{3}(G)} x^{(5 \times 7)^{\alpha}}+\sum_{a b \in E_{4}(G)} x^{(5 \times 8)^{\alpha}} \\
& +\sum_{a b \in E_{5}(G)} x^{(6 \times 7)^{\alpha}}+\sum_{a b \in E_{6}(G)} x^{(6 \times 8)^{\alpha}}+\sum_{a b \in E_{7}(G)} x^{(7 \times 7)^{\alpha}}+\sum_{a b \in E_{8}(G)} x^{(8 \times 8)^{\alpha}} \\
= & \left|E_{1}(G)\right| x^{20^{\alpha}}+\left|E_{2}(G)\right| x^{25^{\alpha}}+\left|E_{3}(G)\right| x^{35^{\alpha}}+\left|E_{4}(G)\right| x^{40^{\alpha}} \\
& +\left|E_{5}(G)\right| x^{42^{\alpha}}+\left|E_{6}(G)\right| x^{48^{\alpha}}+\left|E_{7}(G)\right| x^{49^{\alpha}}+\left|E_{8}(G)\right| x^{64^{\alpha}} \\
= & 8 x^{20^{\alpha}}+(4 \mathrm{~h}+4 \mathrm{k}-8) x^{25^{\alpha}}+(4 \mathrm{~h}+4 \mathrm{k}) x^{35^{\alpha}}+(4 \mathrm{~h}+4 \mathrm{k}-8) x^{40^{\alpha}} \\
& +(4 \mathrm{~h}+4 \mathrm{k}) x^{42^{\alpha}}+(16 \mathrm{hk}-12 \mathrm{~h}-12 \mathrm{k}+8) x^{48^{\alpha}}+(2 \mathrm{~h}+2 \mathrm{k}) x^{49^{\alpha}} \\
& +(16 \mathrm{hk}-8 \mathrm{~h}-8 \mathrm{k}) x^{64^{\alpha}}
\end{aligned}
$$

Which is the required result as shown in equation 2 .

Corollary 3. Replacing $\alpha=1$ and $\alpha=2$ respectively in equation 2 , we get the $M_{2} G_{5}(G, x)$ and $\mathrm{HM}_{2} G_{5}(G, x)$ as follows: 
(i)

$$
\text { (i) } \begin{aligned}
M_{2} G_{5}(G, x)= & (16 \mathrm{hk}-8 \mathrm{~h}-8 \mathrm{k}) x^{64}+(2 \mathrm{~h}+2 \mathrm{k}) x^{49} \\
& +(16 \mathrm{hk}-12 \mathrm{~h}-12 \mathrm{k}+8) x^{48}+(4 \mathrm{~h}+4 \mathrm{k}) x^{42} \\
& +(4 \mathrm{~h}+4 \mathrm{k}-8) x^{40}+(4 \mathrm{~h}+4 \mathrm{k}) x^{35} \\
& +(4 \mathrm{~h}+4 \mathrm{k}-8) x^{25}+8 x^{20} \\
\text { (ii) } \quad H M_{2} G_{5}(G, x)= & (16 \mathrm{hk}-8 \mathrm{~h}-8 \mathrm{k}) x^{4096}+(2 \mathrm{~h}+2 \mathrm{k}) x^{2401} \\
+ & (16 \mathrm{hk}-12 \mathrm{~h}-12 \mathrm{k}+8) x^{2304}+(4 \mathrm{~h}+4 \mathrm{k}) x^{1764} \\
& +(4 \mathrm{~h}+4 \mathrm{k}-8) x^{1600}+(4 \mathrm{~h}+4 \mathrm{k}) x^{1225} \\
& +(4 \mathrm{~h}+4 \mathrm{k}-8) x^{625}+8 x^{400}
\end{aligned}
$$

Proposition 2. Using the first derivative of counting polynomial as shown in equation 2, at $x=1$ we get the general fifth $M_{2}-$ Zagreb index as

(i) $\quad M_{2}^{\alpha} G_{5}(G)=(16 h k-8 h-8 k)(64)^{\alpha}+(2 h+2 k)(49)^{\alpha}$

$$
\begin{aligned}
& +(16 \mathrm{hk}-12 \mathrm{~h}-12 \mathrm{k}+8)(48)^{\alpha}+(4 h+4 k)(42)^{\alpha} \\
& +(4 h+4 k-8)(40)^{\alpha}+(4 \mathrm{~h}+4 \mathrm{k})(35)^{\alpha}+(4 \mathrm{~h}+4 \mathrm{k}-8)(25)^{\alpha} \\
& +8 .(20)^{\alpha} .
\end{aligned}
$$

Corollary 4. From Proposition 2, we get the $M_{2} G_{5}(G)$ and $H M_{2} G_{5}(G)$ by replacing $\alpha=1$ and $\alpha=2$ respectively as follows:

(i) $\quad M_{2} G_{5}(G)=3232 h k-1862 h-1862 k+24$,

(ii) $\quad H M_{2} G_{5}(G)=102400 h k-34758 h-34758 k+3832$.

Theorem 3. The new version of third Zagreb polynomial of $G$ is given by

$$
\begin{aligned}
M_{3} G_{5}(G, x)= & (4 \mathrm{~h}+4 \mathrm{k}-8) \mathrm{x}^{3}+(16 \mathrm{hk}-8 \mathrm{~h}-8 \mathrm{k}+8) x^{2}+(4 h+4 k+8) x \\
& +(16 h k-2 h-2 k-8)
\end{aligned}
$$

Proof. By definition of new version of third Zagreb polynomial index we get,

$$
\begin{aligned}
M_{3} G_{5}(G, x)= & \sum_{a b \in E(G)} x^{\left(\left|S_{G}(a)-S_{G}(b)\right|\right)} \\
= & \sum_{a b \in E_{1}(G)} x^{|(4-5)|}+\sum_{a b \in E_{2}(G)} x^{|(5-5)|}+\sum_{a b \in E_{3}(G)} x^{|(5-7)|}+\sum_{a b \in E_{4}(G)} x^{|(5-8)|} \\
& +\sum_{a b \in E_{5}(G)} x^{|(6-7)|}+\sum_{a b \in E_{6}(G)} x^{|(6-8)|}+\sum_{a b \in E_{7}(G)} x^{|(7-7)|}+\sum_{a b \in E_{8}(G)} x^{|(8-8)|} \\
= & \left|E_{1}(G)\right| x^{1}+\left|E_{2}(G)\right| x^{0}+\left|E_{3}(G)\right| x^{2}+\left|E_{4}(G)\right| x^{3} \\
& +\left|E_{5}(G)\right| x^{1}+\left|E_{6}(G)\right| x^{2}+\left|E_{7}(G)\right| x^{0}+\left|E_{8}(G)\right| x^{0} \\
& =8 x+(4 \mathrm{~h}+4 \mathrm{k}-8)+(4 \mathrm{~h}+4 \mathrm{k}) x^{2}+(4 \mathrm{~h}+4 \mathrm{k}-8) x^{3}+(4 \mathrm{~h}+4 \mathrm{k}) x \\
& +(16 \mathrm{hk}-12 \mathrm{~h}-12 \mathrm{k}+8) x^{2}+(2 \mathrm{~h}+2 \mathrm{k})+(16 \mathrm{hk}-8 \mathrm{~h}-8 \mathrm{k})
\end{aligned}
$$


Hence, the result as in equation 3 .

Proposition 3. Differentiating the counting polynomial as shown in equation 3, with respect to $x$ at $x=1$ we get new version of third Zagreb index $M_{3} G_{5}(G)$ as

(1) $M_{3} G_{5}(G)=32 h k$.

Now we consider line subdivision graph $L(S(G))$ of two dimensional network of benzene ring implanted in $P$-type-surface. The edge partition with respect to the degree sum of neighbor vertices of end vertices of every edge of $L(S(G))$ is shown in Table 2. Total number of edges in $L(S(G))$ is $(88 \mathrm{hk}-10 \mathrm{~h}-10 \mathrm{k})$.

Table 2. The edge partitions with respect to degree sum of neighbor vertices of end vertices of every edge of $L(S(G))$.

\begin{tabular}{cc}
\hline$(\boldsymbol{S}(\boldsymbol{a}), \boldsymbol{S}(\boldsymbol{b})): \boldsymbol{a} \boldsymbol{b} \in \boldsymbol{E}(\boldsymbol{L}(\boldsymbol{S}(\boldsymbol{G})))$ & Total number edges \\
\hline$(4,4)$ & $(4 h+4 k+4)$ \\
$(4,5)$ & $(8 h+8 k+4)$ \\
$(5,5)$ & $(8 \mathrm{hk}-4 \mathrm{~h}-4 \mathrm{k}+4)$ \\
$(5,8)$ & $16 h k$ \\
$(8,8)$ & $(4 h+4 k)$ \\
$(8,9)$ & $(32 h k-8 h-8 k)$ \\
$(9,9)$ & $(32 h k-14 h-14 k)$ \\
\hline
\end{tabular}

Theorem 4. The general fifth $M_{1}$-Zagreb polynomial of $L(S(G))$ is given by

$$
\begin{aligned}
\mathrm{M}_{1}^{\alpha} \mathrm{G}_{5}(\mathrm{~L}(\mathrm{~S}(\mathrm{G})), \mathrm{x})= & (32 h k-14 h-14 k) x^{(18)^{\alpha}}+(32 h k-8 h-8 k) x^{(17)^{\alpha}} \\
& +(4 h+4 k) x^{(16)^{\alpha}}+16 h k \cdot x^{(13)^{\alpha}}+(8 \mathrm{hk}-4 \mathrm{~h}-4 \mathrm{k}+4) x^{(10)^{\alpha}} \\
& +(8 h+8 k+4) x^{9^{\alpha}}+(4 h+4 k+4) x^{8^{\alpha}}
\end{aligned}
$$

Proof. From the definition of general fifth $M_{1}$-Zagreb polynomial index we get,

$$
\begin{aligned}
\mathrm{M}_{1}^{\alpha} \mathrm{G}_{5}(\mathrm{~L}(\mathrm{~S}(\mathrm{G})), \mathrm{x})= & \sum_{a b \in E(\mathrm{~L}(\mathrm{~S}(\mathrm{G}))} x^{\left(S_{G}(a)+S_{G}(b)\right)^{\alpha}} \\
= & \sum_{a b \in E_{1}(\mathrm{~L}(\mathrm{~S}(\mathrm{G}))} x^{(4+4)^{\alpha}}+\sum_{a b \in E_{2}(\mathrm{~L}(\mathrm{~S}(\mathrm{G})))} x^{(4+5)^{\alpha}} \\
+ & \sum_{a b \in E_{3}(\mathrm{~L}(\mathrm{~S}(\mathrm{G})))} x^{(5+5)^{\alpha}}+\sum_{a b \in E_{4}(\mathrm{~L}(\mathrm{~S}(\mathrm{G})))} x^{(5+8)^{\alpha}} \\
+ & \sum_{a b \in E_{5}(\mathrm{~L}(\mathrm{~S}(\mathrm{G})))} x^{(8+8)^{\alpha}}+\sum_{a b \in E_{6}(\mathrm{~L}(\mathrm{~S}(\mathrm{G})))} x^{(8+9)^{\alpha}} \\
+ & \sum_{a b \in E_{7}(\mathrm{~L}(\mathrm{~S}(\mathrm{G})))} x^{(9+9)^{\alpha}} \\
= & \left|E_{1}(\mathrm{~L}(\mathrm{~S}(\mathrm{G})))\right| x^{8^{\alpha}}+\left|E_{2}(\mathrm{~L}(\mathrm{~S}(\mathrm{G})))\right| x^{9^{\alpha}}+\left|E_{3}(\mathrm{~L}(\mathrm{~S}(\mathrm{G})))\right| x^{(10)^{\alpha}} \\
& +\left|E_{4}(\mathrm{~L}(\mathrm{~S}(\mathrm{G})))\right| x^{(13)^{\alpha}}+\left|E_{5}(\mathrm{~L}(\mathrm{~S}(\mathrm{G})))\right| x^{(16)^{\alpha}} \\
& +\left|E_{6}(\mathrm{~L}(\mathrm{~S}(\mathrm{G})))\right| x^{(17)^{\alpha}}+\left|E_{7}(\mathrm{~L}(\mathrm{~S}(\mathrm{G})))\right| x^{(18)^{\alpha}} \\
= & (4 h+4 k+4) x^{8^{\alpha}}+(8 h+8 k+4) x^{9^{\alpha}}+(8 \mathrm{hk}-4 \mathrm{~h}-4 \mathrm{k}+4) x^{(10)^{\alpha}}
\end{aligned}
$$




$$
\begin{aligned}
& +16 h k \cdot x^{(13)^{\alpha}}+(4 h+4 k) x^{(16)^{\alpha}}+(32 h k-8 h-8 k) x^{(17)^{\alpha}} \\
& +(32 h k-14 h-14 k) x^{(18)^{\alpha}}
\end{aligned}
$$

Which is the desired result, as shown in equation 4.

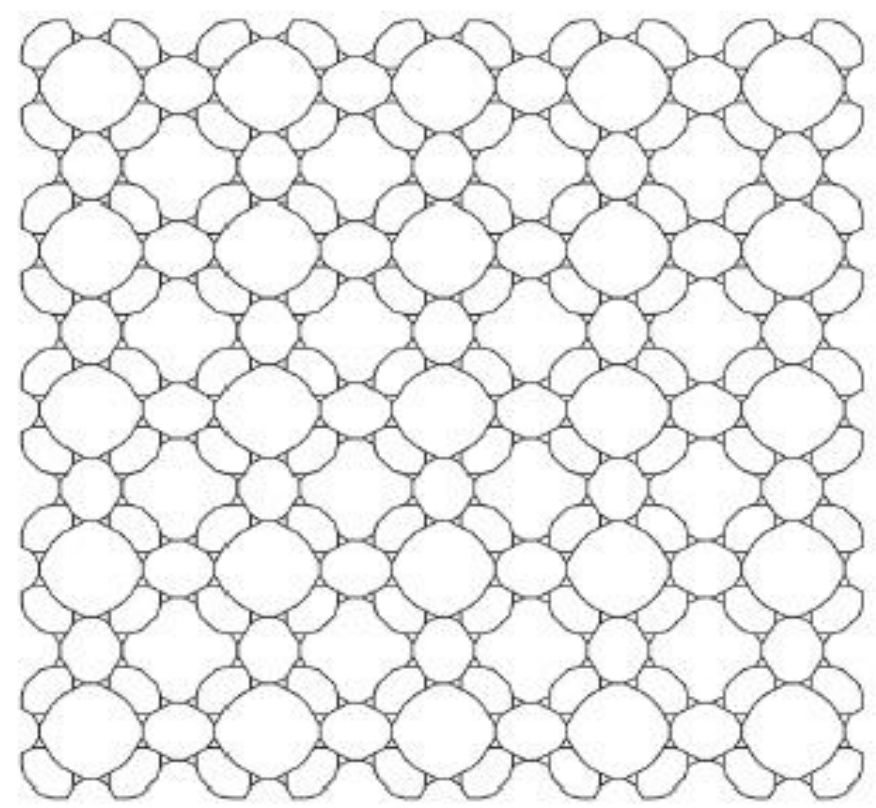

Figure 2. Line subdivision graph of benzene ring implanted in P-type-surface network

Corollary 5. Replacing $\alpha=1$ and $\alpha=2$, respectively in equation 4, we get $M_{1} G_{5}(L(S(G)), x)$ and $H M_{1} G_{5}(L(S(G)), x)$ as follows:

(i)

$$
\text { (i) } \begin{aligned}
M_{1} G_{5}(L(S(G)), x)= & (32 h k-14 h-14 k) x^{18}+(32 h k-8 h-8 k) x^{17} \\
& +(4 h+4 k) x^{16}+16 h k \cdot x^{13}+(8 h \mathrm{k}-4 \mathrm{~h}-4 \mathrm{k}+4) x^{10} \\
& +(8 h+8 k+4) x^{9}+(4 h+4 k+4) x^{8} \\
\text { (ii) } H M_{1} G_{5}(L(S(G)), x)= & (32 h k-14 h-14 k) x^{324}+(32 h k-8 h-8 k) x^{289} \\
& +(4 h+4 k) x^{256}+16 h k \cdot x^{169} \\
& +(8 \mathrm{hk}-4 \mathrm{~h}-4 \mathrm{k}+4) x^{100}+(8 h+8 k+4) x^{81} \\
& +(4 h+4 k+4) x^{64} .
\end{aligned}
$$

Proposition 4. Differentiate the counting polynomial as shown in equation 4, with respect to $x$ at $x=1$, we get the general fifth $M_{1}$-Zagreb index of $L(S(G))$ as follows:

$$
\begin{aligned}
\mathrm{M}_{1}^{\alpha} \mathrm{G}_{5}(L(S(G))) & =(32 h k-14 h-14 k)(18)^{\alpha}+(32 h k-8 h-8 k)(17)^{\alpha} \\
& +(4 h+4 k) x^{(16)^{\alpha}}+16 h k \cdot x^{(13)^{\alpha}}+(8 \mathrm{hk}-4 \mathrm{~h}-4 \mathrm{k}+4) x^{(10)^{\alpha}} \\
& +(8 h+8 k+4) x^{9^{\alpha}}+(4 h+4 k+4) x^{8^{\alpha}}
\end{aligned}
$$

Corollary 6. Replacing $\alpha=1$ and $\alpha=2$ respectively in Proposition 4, we get $M_{1} G_{5}(L(S(G)))$ and $H M_{1} G_{5}(L(S(G)))$ as follows: 
(iii) $M_{1} G_{5}(L(S(G)))=1408 h k-260 h-260 k+108$,

(iv) $H M_{1} G_{5}(L(S(G)))=23120 h k-5320 h-5320 k+980$.

Theorem 5. The general fifth $M_{2}$-Zagreb polynomial of $L(S(G))$ is given by $\mathrm{M}_{2}^{\alpha} \mathrm{G}_{5}(\mathrm{~L}(\mathrm{~S}(\mathrm{G})), \mathrm{x})=(32 h k-14 h-14 k) x^{(81)^{\alpha}}+(32 h k-8 h-8 k) x^{(72)^{\alpha}}$

$$
\begin{aligned}
& +(4 h+4 k) x^{(64)^{\alpha}}+16 h k \cdot x^{(40)^{\alpha}}+(8 \mathrm{hk}-4 \mathrm{~h}-4 \mathrm{k}+4) x^{(25)^{\alpha}} \\
& +(8 h+8 k+4) x^{(20)^{\alpha}}+(4 h+4 k+4) x^{(16)^{\alpha}}
\end{aligned}
$$

Proof. From the definition of general fifth $M_{2}$-Zagreb polynomial index we get,

$$
\begin{aligned}
\mathrm{M}_{2}^{\alpha} \mathrm{G}_{5}(\mathrm{~L}(\mathrm{~S}(\mathrm{G})), \mathrm{x})= & \sum_{a b \in E(\mathrm{~L}(\mathrm{~S}(\mathrm{G}))} x^{\left(S_{G}(a) S_{G}(b)\right)^{\alpha}} \\
= & \sum_{a b \in E_{1}(\mathrm{~L}(\mathrm{~S}(\mathrm{G})))} x^{(4 \times 4)^{\alpha}}+\sum_{a b \in E_{2}(\mathrm{~L}(\mathrm{~S}(\mathrm{G})))} x^{(4 \times 5)^{\alpha}} \\
& +\sum_{a b \in E_{3}(\mathrm{~L}(\mathrm{~S}(\mathrm{G})))} x^{(5 \times 5)^{\alpha}}+\sum_{a b \in E_{4}(\mathrm{~L}(\mathrm{~S}(\mathrm{G})))} x^{(5 \times 8)^{\alpha}} \\
& +\sum_{a b \in E_{5}(\mathrm{~L}(\mathrm{~S}(\mathrm{G})))} x^{(8 \times 8)^{\alpha}}+\sum_{a b \in E_{6}(\mathrm{~L}(\mathrm{~S}(\mathrm{G})))} x^{(8 \times 9)^{\alpha}} \\
& +\sum_{a b \in E_{7}(\mathrm{~L}(\mathrm{~S}(\mathrm{G})))} x^{(9 \times 9)^{\alpha}} \\
= & \left|E_{1}(\mathrm{~L}(\mathrm{~S}(\mathrm{G})))\right| x^{(16)^{\alpha}}+\left|E_{2}(\mathrm{~L}(\mathrm{~S}(\mathrm{G})))\right| x^{(20)^{\alpha}} \\
& +\left|E_{3}(\mathrm{~L}(\mathrm{~S}(\mathrm{G})))\right| x^{(25)^{\alpha}}+\left|E_{4}(\mathrm{~L}(\mathrm{~S}(\mathrm{G})))\right| x^{(40)^{\alpha}} \\
& +\left|E_{5}(\mathrm{~L}(\mathrm{~S}(\mathrm{G})))\right| x^{(64)^{\alpha}}+\left|E_{6}(\mathrm{~L}(\mathrm{~S}(\mathrm{G})))\right| x^{(72)^{\alpha}} \\
& +\left|E_{7}(\mathrm{~L}(\mathrm{~S}(\mathrm{G})))\right| x^{(81)^{\alpha}} \\
= & (4 h+4 k+4) x^{(16)^{\alpha}}+(8 h+8 k+4) x^{(20)^{\alpha}} \\
& +(8 h \mathrm{~h}-4 \mathrm{~h}-4 \mathrm{k}+4) x^{(25)^{\alpha}}+16 h k \cdot x^{(40)^{\alpha}}+(4 h+4 k) x^{(64)^{\alpha}} \\
& +\left(32 h k-8 h-8 k x^{(72)^{\alpha}}+(32 h k-14 h-14 k) x^{(81)^{\alpha}}\right.
\end{aligned}
$$

Hence the result.

Corollary 7. Putting $\alpha=1$ and $\alpha=2$ respectively in equation 5 , we get $M_{2} G_{5}(L(S(G)), x)$ and $H M_{2} G_{5}(L(S(G)), x)$ as follows:

(i) $\quad M_{2} G_{5}(L(S(G)), x)=(32 h k-14 h-14 k) x^{(81)}+(32 h k-8 h-8 k) x^{(72)}$

$$
\begin{aligned}
& +(4 h+4 k) x^{(64)}+16 h k \cdot x^{(40)}+(8 \mathrm{hk}-4 \mathrm{~h}-4 \mathrm{k}+4) x^{(25)} \\
& +(8 h+8 k+4) x^{(20)}+(4 h+4 k+4) x^{(16)}
\end{aligned}
$$


(ii)

$$
\begin{aligned}
H M_{2} G_{5}(L(S(G)), x)= & (32 h k-14 h-14 k) x^{(6561)} \\
& +(32 h k-8 h-8 k) x^{(5184)}+(4 h+4 k) x^{(4096)} \\
& +16 h k \cdot x^{(1600)}+(8 \mathrm{hk}-4 \mathrm{~h}-4 \mathrm{k}+4) x^{(625)} \\
& +(8 h+8 k+4) x^{(400)}+(4 h+4 k+4) x^{(256)}
\end{aligned}
$$

Proposition 5. Appling the first derivative of counting polynomial as shown in equation 5, at $x=1$, we get the general fifth $M_{2}$-Zagreb index of $L(S(G))$ as

(i) $\quad \mathrm{M}_{2}^{\alpha} \mathrm{G}_{5}(L(S(G)))=(32 h k-14 h-14 k)(81)^{\alpha}+(32 h k-8 h-8 k)(72)^{\alpha}$

$$
\begin{aligned}
& +(4 h+4 k)(64)^{\alpha}+16 h k(40)^{\alpha}+(8 \mathrm{hk}-4 \mathrm{~h}-4 \mathrm{k}+4)(25)^{\alpha} \\
& +(8 h+8 k+4)(20)^{\alpha}+(4 h+4 k+4)(16)^{\alpha}
\end{aligned}
$$

Corollary 8. We compute the $M_{2} G_{5}(L(S(G)))$ and $H M_{2} G_{5}(L(S(G)))$ by replacing $\alpha=$ 1 and $\alpha=2$ respectively in Proposition 5, as follows:

(i) $\quad M_{2} G_{5}(L(S(G)))=5736 h k-1330 h-1330 k+244$,

(ii) $\quad H M_{2} G_{5}(L(S(G)))=406440 h k-115218 h-115218 k+5124$.

Theorem 6. The new version of third Zagreb polynomial of $L(S(G))$ is given by

$M_{3} G_{5}(L(S(G)), x)=16 h k x^{3}+(32 h k+4) x+(40 h k-10 h-10 k+8)$.

Proof. From the definition of new version of third Zagreb polynomial index we get,

$$
\begin{aligned}
\mathrm{M}_{3} \mathrm{G}_{5}(\mathrm{~L}(\mathrm{~S}(\mathrm{G})), \mathrm{x})= & \sum_{a b \in E(\mathrm{~L}(\mathrm{~S}(\mathrm{G}))} x^{\left(\left|S_{G}(a)-S_{G}(b)\right|\right)} \\
= & \sum_{a b \in E_{1}(\mathrm{~L}(\mathrm{~S}(\mathrm{G}))} x^{(|4-4|)}+\sum_{a b \in E_{2}(\mathrm{~L}(\mathrm{~S}(\mathrm{G})))} x^{(|4-5|)} \\
& +\sum_{a b \in E_{3}(\mathrm{~L}(\mathrm{~S}(\mathrm{G})))} x^{(|5-5|)}+\sum_{a b \in E_{4}(\mathrm{~L}(\mathrm{~S}(\mathrm{G})))} x^{(|5-8|)} \\
& +\sum_{a b \in E_{5}(\mathrm{~L}(\mathrm{~S}(\mathrm{G})))} x^{(|8-8|)}+\sum_{a b \in E_{6}(\mathrm{~L}(\mathrm{~S}(\mathrm{G})))} x^{(|8-9|)} \\
& +\sum_{a b \in E_{7}(\mathrm{~L}(\mathrm{~S}(\mathrm{G})))} x^{(|9-9|)} \\
& =\left|E_{1}(\mathrm{~L}(\mathrm{~S}(\mathrm{G})))\right| x^{0}+\left|E_{2}(\mathrm{~L}(\mathrm{~S}(\mathrm{G})))\right| x^{1}+\left|E_{3}(\mathrm{~L}(\mathrm{~S}(\mathrm{G})))\right| x^{0} \\
& +\left|E_{4}(\mathrm{~L}(\mathrm{~S}(\mathrm{G})))\right| x^{3}+\left|E_{5}(\mathrm{~L}(\mathrm{~S}(\mathrm{G})))\right| x^{0}+\left|E_{6}(\mathrm{~L}(\mathrm{~S}(\mathrm{G})))\right| x^{1}
\end{aligned}
$$




$$
\begin{gathered}
+\left|E_{7}(\mathrm{~L}(\mathrm{~S}(\mathrm{G})))\right| x^{0} \\
=(4 h+4 k+4) x^{0}+(8 h+8 k+4) x+(8 \mathrm{hk}-4 \mathrm{~h}-4 \mathrm{k}+4) x^{0}+16 h k \cdot x^{3} \\
+(4 h+4 k) x^{0}+(32 h k-8 h-8 k) x+(32 h k-14 h-14 k) x^{0}
\end{gathered}
$$

Hence the result follows as in equation 6 .

Proposition 6. The first derivative of counting polynomial as shown in equation 6 , with respect to $x$ at $x=1$ we get $M_{3} G_{5}(L(S(G)))$ as

$$
\text { (i) } M_{3} G_{5}(L(S(G)))=80 h k+4
$$

\section{Conclusions}

In this work, we computed general fifth $M-$ Zagreb polynomials and the new version of third Zagreb polynomial for the molecular graph $G$ of the two-dimensional network of benzene ring implanted in the $P$-type surface and its line subdivision graph $L(S(G))$. Hence we computed some other polynomials and their corresponding topological indices such as fifth $M$-Zagreb polynomials and their corresponding fifth $M$-Zagreb indices, fifth hyper $M$-Zagreb polynomials and their corresponding fifth hyper $M-$ Zagreb indices, $M_{3}$-Zagreb polynomial and it is corresponding $M_{3}$-Zagreb index by using our derived results. In the future study, we want to compute these indices for some chemically important molecular structures.

\section{Funding}

This research was funded by the COUNCIL OF SCIENTIFIC AND INDUSTRIAL RESEARCH (CSIR), grant number 09/973(0016)/2017-EMR-I.

\section{Acknowledgments}

We would like to thank the Editor in Chief of the journal as well as the anonymous reviewers for their careful reading of our manuscript and their many insightful comments and suggestions to improve our manuscript.

\section{Conflicts of Interest}

The authors declare no conflict of interest.

\section{References}

1. Abdelgader, M. S.; Wang, C.; Mohammed, S.A. Computation of Topological Indices of Some Special Graphs. Mathematics 2018, 6, 1-15, https://doi.org/10.3390/math6030033.

2. Liu, J. B.; Shafiq, K.M.; Ali, H.; Naseem, A.; Maryam, N.; Asghar, S.S. Topological Indices of mth Chain Silicate Graphs. Mathematics 2019, 7, 1-16, https://doi.org/10.3390/math7010042.

3. Ma, Y.; Cao, S.; Shi, Y.; Gutman, I.; Dehmer, M.; Furtula, B. From the Connectivity Index to Various Randić-Type Descriptors. MATCH Commun. Math. Comput. Chem. 2018, 80, 85-106.

4. Manimekalai, S.; Mary, U.; Lavanya, M. Computation of topological indices using python program for chemical graph structure. Journal of Physics: Conference Series 2018, 1139, 1-8, https://doi.org/10.1088/1742-6596/1139/1/012060. 
5. Gutman, I.; Milovanović, E.; Milovanović, I. Beyond the Zagreb indices. AKCE International Journal of Graphs and Combinatorics 2018, https://doi.org/10.1016/j.akcej.2018.05.002.

6. Gao, W.; Iqbal, Z.; Ishaq, M.; Sarfraz, R.; Aamir, M.; Aslam, A. On Eccentricity-Based Topological Indices Study of a Class of Porphyrin-Cored Dendrimers. Biomolecules 2018, 8, https://doi.org/10.3390/biom8030071

7. Shin, K.M.; Manzoor, Z.A.; Rehamn, V.A.; Waqas, N.; Wei, G. Calculating the Degree-based Topological Indices of Dendrimers. Open Chemistry 2018, 16, 681-688, https://doi.org/10.1515/chem-2018-0071

8. Shailaja, S.; Manjula, K.; Hosamani, S.M. QSPR Analysis of Certain Distance Based Topological Indices. Applied Mathematics and Nonlinear Sciences 2019, 4, 371-386, https://doi.org/10.2478/AMNS.2019.2.00032.

9. Asok, A.; Kureethara, V.J. QSPR Analysis of certain degree and eccentricity based topological indices and butane derivatives. Rasayan Journal of Chemistry 2019, 12, 464-470, http://dx.doi.org/10.31788/RJC.2019.1225093.

10. Mekenyan, O.; Bonchev, D.; Sabljic, A.; Trinajstić, N. Applications of Topological Indices to QSAR. The Use of the Balaban Index and the Electropy Index for Correlations with Toxicity of Ethers on Mice. Acta pharmaceutica Jugoslavica 1987, 37, 75-86.

11. Pyka, A. Application of topological indices for prediction of the biological activity of selected alkoxyphenols. Acta Poloniae Pharmaceutica 2002, 59, 347-351.

12. Graovac, A.; Ghorbani, M.; Hosseinzadeh, M.A. Computing fifth geometric-arithmetic index for nanostar dendrimers. Journal of Mathematical Nanoscience 2011, 1, 33-42, https://doi.org/10.22061/jmns.2011.461.

13. Kulli, V. General fifth M-Zagreb indices and fifth M-Zagreb polynomials of dendrimer nanostars. International Journal of Fuzzy Mathematical Archive 2017, 13, 99-103, http://doi.org/10.22457/ijfma.v13n1a10.

14. Nadeem, M.; Yousaf, A.; Alolaiyan, H.; Razaq, A. Certain polynomials and related topological indices for the series of benzenoid graphs. Scientific Reports 2019, 9, https://doi.org/10.1038/s41598-019-45721-y.

15. Kulli, V.R. Reduced second hyper-Zagreb index and its polynomial of certain silicate networks. Journal of Mathematics and Informatics 2018, 14, 11-16, http://dx.doi.org/10.22457/jmi.v14a2.

16. Baig, A.Q.; Naeem, M.; Gao, W.; Liu, J.B. General fifth M-Zagreb indices and fifth M-Zagreb polynomials of carbon graphite. Eurasian Chemical Communications 2020, 2, 634-640, https://doi.org/10.33945/SAMI/ECC.2020.5.10.

17. Shin, K.M.; Muhammad, Y.; Manzoor, Z.A.; Muhammad, Y.; Waqas, N. Zagreb polynomials and redefined Zagreb indices of nanostar dendrimers. Open Physics 2019, 17, 31-40, https://doi.org/10.1515/phys-20190004.

18. Shao, Z.; Siddiqui, K.M.; Muhammad, H.M. Computing Zagreb Indices and Zagreb Polynomials for Symmetrical Nanotubes. Symmetry 2018, 10, 1-16. https://doi.org/10.3390/sym10070244.

19. Deutsch, E.; Klavžar, S. On the M-polynomial of planar chemical graphs. Iranian Journal of Mathematical Chemistry 2020, 11, 65-71.

20. Ikonen, T.; Kalidas, N.; Lahtinen, K.; Isoniemi, T.; Toppari, J.J.; Vázquez, E.; Herrero-Chamorro, M.A.; Fierro, J.L.G.; Kallio, T.; Lehto, V.P. Conjugation with carbon nanotubes improves the performance of mesoporous silicon as Li-ion battery anode. Scientific Reports 2020, 10, https://doi.org/10.1038/s41598-02062564-0.

21. Blien, S.; Steger, P.; Hüttner, N.; Graaf, R.; Hüttel, A.K. Quantum capacitance mediated carbon nanotube optomechanics. Nature Communications 2020, 11, https://doi.org/10.1038/s41467-020-15433-3.

22. Jagdale, S.; Bafna, M.; Chabukswar, A. Transdermal delivery of solid lipid nanoparticles of ketoprofen for treatment of arthritis. Letters in Applied NanoBioScience 2019, 8, 627-634, https://doi.org/10.33263/LIANBS83.627636.

23. Mathiyalagan, S.; Mandal, B.K. Preparation of metal doped quercetin nanoparticles, characterization and their stability study. Letters in Applied NanoBioScience 2019, 8, 704-710, https://doi.org/10.33263/LIANBS84.704710.

24. Mackay, A.L.; Terrones, H. Diamond from graphite. Nature 1991, 352, 762-762, https://doi.org/10.1038/352762a0.

25. Lenosky, T.; Gonze, X.; Teter, M.; Elser, V. Energetics of negatively curved graphitic carbon. Nature 1992, 355, 333-335, https://doi.org/10.1038/355333a0.

26. Keeffe, M.O.; Adams, G.B.; Sankey, O.F. Predicted new low energy forms of carbon. Physical Review Letters 1992, 68, 2325-2328, https://doi.org/10.1103/PhysRevLett.68.2325.

27. Ahmad, A.; Elahi, K.; Hasni, R.; Nadeem, M.F. Computing the degree based topological indices of line graph of benzene ring embedded in P-type-surface in 2D network. Journal of Information and Optimization Sciences 2019, 40, 1511-1528, https://doi.org/10.1080/02522667.2018.1552411. 